\title{
Performance Analysis of U-FBG Fiber System using different Sizes of Bragg Cell
}

\author{
Manjinder Kaur \\ Department of Electronics \& Communication \\ Engineering \\ Shaheed Bhagat Singh State Technical Campus \\ Ferozepur 152004, Punjab, India
}

\author{
Sanjeev Dewra, PhD \\ Department of Electronics \& Communication \\ Engineering \\ Shaheed Bhagat Singh State Technical Campus \\ Ferozepur 152004, Punjab, India
}

\begin{abstract}
In practical applications, the Uniform fiber Bragg grating (UFBG) is a key component of integrated photonic circuits like optical filter, splitters, optical sensor and switches etc. This paper evaluates the performance of U-FBG fiber system by changing the size of Bragg cell based on Surface plasmon polaritons (SPP) using silver (Ag) profile material by Finite Difference Time domain (FDTD) method. It is observed that the maximum received optical power at the output port achieved is $8.48 \times 10^{-4} \mathrm{w} / \mathrm{m}^{2}$ with $0.9 \mu \mathrm{m}$ radius of bragg cell and Silver $(\mathrm{Ag})$ profile material at $0.8 \mathrm{w} / \mathrm{m}^{2}$ input transmission power whereas at $1.2 \mu \mathrm{m}$ radius of bragg cell have low output power of $7.56 \times 10^{-4} \mathrm{w} / \mathrm{m}^{2}$.
\end{abstract}

\section{Keywords}

Uniform Fiber Bragg Grating (U-FBG), Wavelength-divisionmultiplexing (WDM), Chirped Fiber Bragg gratings (CFBGs), Vestigial sideband (VSB), Arrayed waveguide grating (AWG).

\section{INTRODUCTION}

In the designing field of fiber optic sensors Fiber Bragg gratings (FBG) have a major role in its potential for the measurement of several important physical magnitudes such as temperature, strain etc. The suitable design strategies must be adopted to make simultaneous measurement with minimum cross-sensitivity for both temperature and strain [1]. In all fiber based devices, FBGs play a key role as components due to their advantageous features such as low insertion loss, simplicity, low cost, polarization independence and seamless integration in fiber optic systems. Moreover, FBGs are immune to electromagnetic interference (EMI), non-conducting, chemically inert and spark free as they composed of dielectric material [2]. For these reasons FBGs have been extensively implemented in different kinds of application scenarios such as sensors [3], filters [4], switches [5], and for multiplying pulse repetition rates [6], amongst others. FBG comprises of a periodic refractive index modulation in the core of a single mode optical fiber, in which the phase fronts are perpendicular to the longitudinal axis of the fiber, also the accurate and direct measurement of gratings period can be done by using optical imaging technique [7]. Fiber Bragg gratings (FBGs) are promising sub-elements in a range of network components because of their potential low cost [8]. For maximum bandwidth usage and resilience to laser wavelength drift, a fiber Bragg grating filter should have a square-topped profile with steep sides in reflection, low side lobes and flat dispersion. Advanced FBGs with very sharp reflectivity edges and required time delay characteristics in the pass band can be fabricated [9]. M. M. Ali et.al [10], demonstrated an optical notch filter constructed from a periodic micro bent fiber Bragg grating attained by using two copper-wire-wound slabs. In the reflection spectrum, sideband peaks are created as a result of mechanically induced modulation on the grating period, and a higher number of peaks are observed when greater modulation is applied. The peak wavelength spacing depends upon the period of micro bending, which can be varied by changing the diameter of the winding wire or the angle of placement of fiber Bragg grating placed in between the two wound slabs. P. Niewczas et.al [11] proposed an architecture of the interrogation system of fiber Bragg grating (FBG) which is dependent on an arrayed waveguide grating (AWG) device. By using an AWG the spectrum of reflected light is analyzed from the FBG sensor, which appears as a coarse -spectrometer. E.G. Turitsyna et.al [12] proposed a basic design with low dispersion and sharp reflection of non-uniform FBG (fiber Bragg grating) and the effect on the performance of transmission system of ultrasquare FBG-based filters for different design solutions is investigated.

In this paper we proposed and analysis the performance of $U$ FBG fiber by changing the radius the of bragg cell using silver (Ag) profile material based on Surface plasmon polaritons (SPP) by using Finite Differences Time domain (FDTD) method to simulate and analysis of new optical properties. The previous work [12] was carried out for designed a plasmonic Bragg gratings based on a plasmonic trench waveguide using silver profile material at $1410 \mathrm{~nm}$ input transmission wavelength. The work is extended here for designed a U-FBG fiber based on SPP using silver (Ag) profile material with minimum input transmission power from $0.1 \mathrm{w} / \mathrm{m}^{2}$ to $0.8 \mathrm{w} / \mathrm{m}^{2}$ at $1330 \mathrm{~nm}$ and $1550 \mathrm{~nm}$ input transmission wavelength using FDTD.

After introduction in section 1, a proposed system configuration is presented in Section 2. Further section 3 displays the results and discussion and finally the conclusion is drawn in Section 4.

\section{SYSTEM CONFIGURATION OF U- FBG}

The proposed structure of Uniform Fiber Bragg Grating fiber is based on SPP is designed as shown in Fig.1.The architecture of Uniform Fiber Bragg Grating fiber consists of two linear waveguide and six circular waveguide's. The core and cladding area of fiber are designed by using two linear waveguides based on silver $(\mathrm{Ag})$ dopant material profile with refractive indexes of 2.41. The refractive index of core is more than the refractive index of cladding. The cross section view of the U-FBG fiber is shown in Fig. 1. The linear waveguide of properties are: width is (W) $0.3 \mu \mathrm{m}$ and length of waveguide is (L) $22 \mu \mathrm{m}$. 


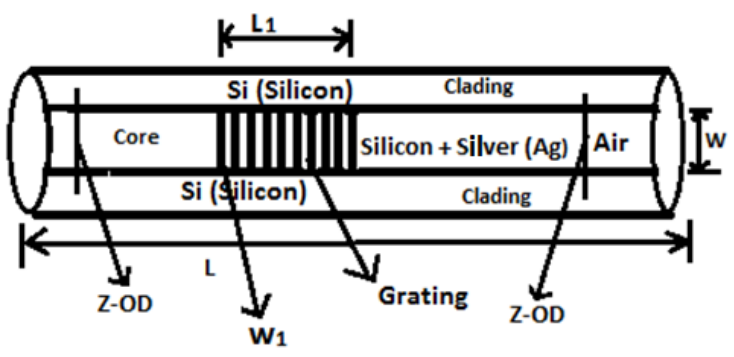

Figure.1. System Configuration of U-FBG

The six circular waveguides are used for design the grating in fiber. The radius $\left(\mathrm{R}_{1}\right)$ of circular waveguides is changed from $0.9 \mu \mathrm{m}$ to $1.2 \mu \mathrm{m}$. The Modulated CW Laser is used as input optical source with wavelength of $1.33 \mu \mathrm{m}$ and $1.55 \mu \mathrm{m}$ at input transmission powers from $0.1 \mathrm{w} / \mathrm{m}^{2}$ to $0.8 \mathrm{w} / \mathrm{m}^{2}$. The input Field Properties of input optical source is 2.02 of modal indexes with modal field transverse. Due to the large optical field enhancement and confinement of metallic structures, the structure of U-FBG fiber based on SPPs is proposed. In the 2D structure shown in Fig. 2, the dopants metal supporting for design the Uniform grating in fiber is assumed to be Silver $(\mathrm{Ag})$, whose refractive index is 2.41 for $1.33 \mu \mathrm{m}$ and $1.55 \mu \mathrm{m}$ input transmission wavelength. The 2D-FDTD method is implemented to simulate the behavior of the device.

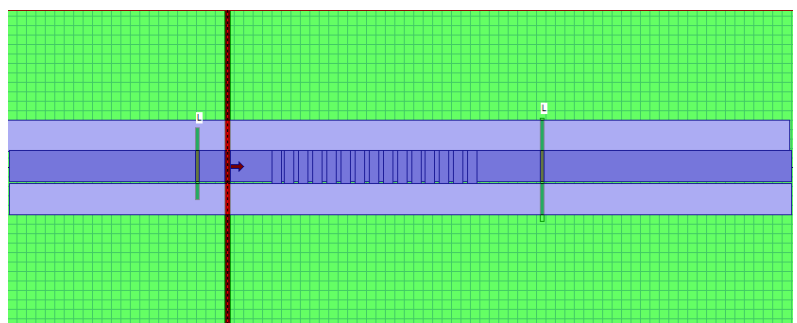

Figure.2.2D structure of U-FBG Fiber

Hence to block specific wavelengths in fiber, FBG can be used as an optical inline filter or a wavelength-specific reflector. The two Z-direction Line optical detectors (OD) are used at the output port and input port (Reflected port) of UFBG as shown in Fig.1. The two point optical detectors are used for measuring the normalized optical power at the reflected port and output port.

\section{RESULTS AND DISCUSSION}

This paper present the performance of U-FBG fiber by varying the shape of bragg cell using Silver (Ag) profile material based on SPP using FDTD method to simulate and analysis of new optical properties. A fiber Bragg grating (FBG) is a type of distributed Bragg reflector constructed in a short segment of optical fiber that reflects certain wavelengths of light and transmits all others. This is achieved by creating a periodic variation in the refractive index of the fiber core, radius of bragg cell, grating period and length of grating, which generates a wavelength-specific dielectric mirror. The numerical calculations clarified the power at each port after the light has reached a steady state and evaluated by using the two Z-line optical detectors at the reflected port and output port of UFBG fiber. The Bragg wavelength is a tuned wavelength and reflected from U-FBG fiber and hence written as: [13],

$\kappa_{\mathrm{B}}=2 \mathrm{n}_{\mathrm{e}} \Lambda$

Where, $\kappa_{\mathrm{B}}=$ Bragg wavelength $\mathrm{n}_{\mathrm{e}}=$ effective refractive index of grating in the fiber core

$\Lambda=$ grating Period.

In U-FBG fiber optical signal reflectivity is a function of detuning length of grating and effective index of grating as:

$\mathrm{R}_{\mathrm{FBG}}=\mathrm{r}(\mathrm{W})=\frac{i \cdot k \sin (q \cdot L g)}{\cos (q \cdot L g)-i \cdot \delta \cdot \sin (q . L g)}$

$\mathrm{L}_{\mathrm{g}}=$ grating length of T-FBG

$\delta=\frac{2 \pi}{\lambda_{o}}-\frac{2 \pi}{\lambda_{B}}$

$\mathrm{K}=\frac{\pi \cdot \mathrm{n}_{\mathrm{g}} \cdot \Gamma}{\lambda_{\mathrm{B}}}$

$\mathrm{k}=$ coefficient of coupling

$\mathrm{n}_{\mathrm{g}}=$ effective refractive index of grating

$\Gamma=$ confinement factor

$\lambda_{\mathrm{B}}=$ Bragg wavelength.

The change in grating length, grating effective index and apodization than optical signal reflectivity is varied through U-FBG and we know that the optical signal reflectivity is function of these parameters as written in Eq. 2 .

Figure.3. depicts the received optical power versus input transmission power at $0.9 \mu \mathrm{m}$ radius of bragg cell by using sliver $(\mathrm{Ag})$ profile material at $1.55 \mu \mathrm{m}$ input transmission wavelength. It is clear from the plot that as the input transmission power varies from $0.1 \mathrm{w} / \mathrm{m}^{2}$ to $0.8 \mathrm{w} / \mathrm{m}^{2}$ then the received optical power is also changed at both i.e. output port and reflected port. The variation of received optical power at both ports is function of grating period and refractive index which is changed due to variation in the radius of bragg cell.

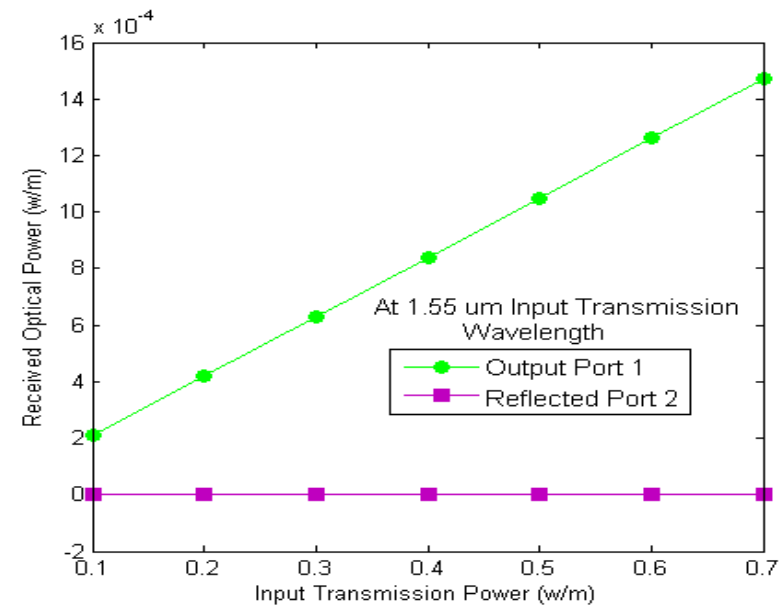

Figure.3. Received Optical Power $\left(\mathrm{w} / \mathrm{m}^{2}\right)$ versus input transmission power $\left(\mathrm{w} / \mathrm{m}^{2}\right)$ at $0.9 \mu \mathrm{m}$ radius of bragg cell of U-FBG Fiber 


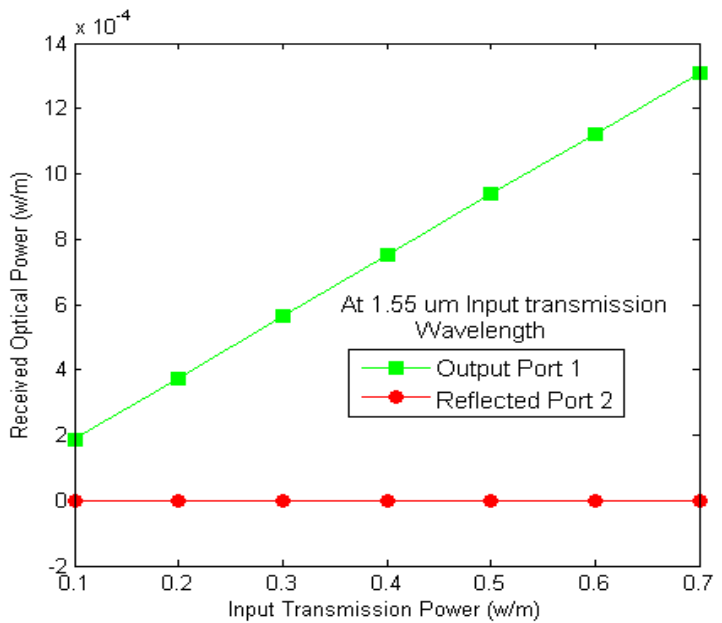

Figure.4. Received Optical Power $\left(\mathrm{w} / \mathrm{m}^{2}\right)$ versus input transmission power $\left(\mathrm{w} / \mathrm{m}^{2}\right)$ at $1.2 \mathrm{um}$ radius of bragg cell of U-FBG fiber.

Figure. 4, shows the Plot of received Optical power $w / \mathrm{m}^{2}$ versus input transmission power $\mathrm{w} / \mathrm{m}^{2}$ at $1.2 \mu \mathrm{m}$ radius of bragg cell by using silver (Ag) profile material at $1.55 \mu \mathrm{m}$ input transmission wavelength of U-FBG fiber. When the input transmission power is varied from $0.1 \mathrm{w} / \mathrm{m}^{2}$ to $0.8 \mathrm{w} / \mathrm{m}^{2}$ then the received optical power is also varied at the output port and reflected port of U-FBG fiber. As the radius of bragg cell is changed from $0.9 \mu \mathrm{m}$ to $1.2 \mu \mathrm{m}$ then the received optical power is also changed.

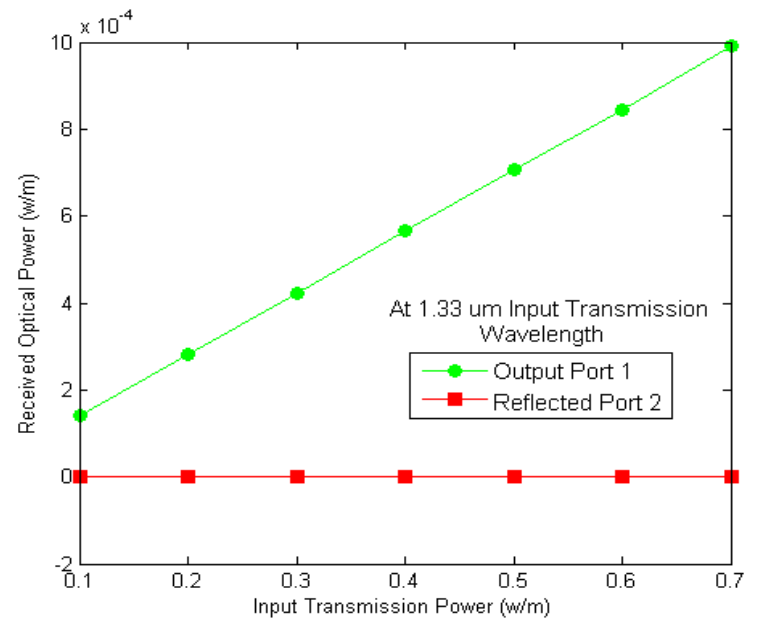

Figure.5. Received Optical Power $\left(\mathrm{w} / \mathrm{m}^{2}\right)$ versus input transmission power $\left(\mathrm{w} / \mathrm{m}^{2}\right)$ at $0.9 \mathrm{um}$ radius of bragg cell of U-FBG fiber.

Figure .5 , shows the received Optical power $\left(\mathrm{w} / \mathrm{m}^{2}\right)$ versus input transmission power $\left(\mathrm{w} / \mathrm{m}^{2}\right)$ at $0.9 \mu \mathrm{m}$ radius of bragg cell by using silver (Ag) profile material at $1.33 \mu \mathrm{m}$ input transmission wavelength of U-FBG fiber. The received optical power is varied at the both output port and reflected port due to variation of input transmission power from $0.1 \mathrm{w} / \mathrm{m}^{2}$ to 0.8 $\mathrm{w} / \mathrm{m}^{2}$.

Figure.6. depicts the received optical power versus input transmission power at $1.2 \mu \mathrm{m}$ radius of bragg cell by using silver (Ag) profile material at $1.33 \mu \mathrm{m}$ input transmission wavelength of U-FBG. It is cleared from plot as the input transmission power is changed from $0.1 \mathrm{w} / \mathrm{m}^{2}$ to $0.8 \mathrm{w} / \mathrm{m}^{2}$ then the received optical power is also changed at both output port and reflected port. The variation of received optical power at both ports is function of grating period and refractive index which is changed due to variation in the radius of bragg cell.

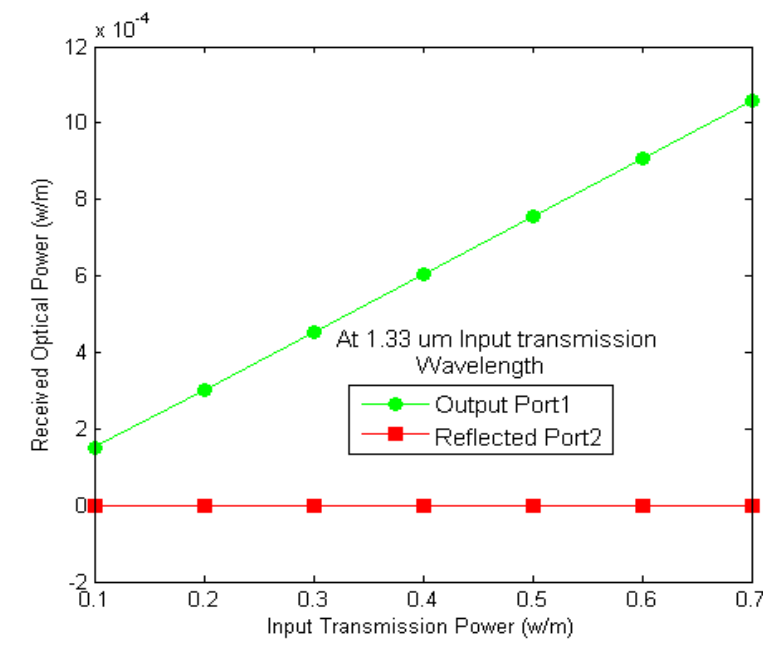

Figure.6. Received Optical Power w/ $\mathrm{m}^{2}$ versus input transmission power $w / \mathrm{m}^{2}$ at $1.2 \mu \mathrm{m}$ radius of bragg cell of U-FBG fiber.

\section{CONCLUSION}

This paper presents the performance of U-FBG fiber by changing the size of bragg cell based on SPP using Finite Differences Time domain method is simulated and analysis of new optical properties by using Sliver $(\mathrm{Ag})$ profile material. The simulation results show that the Silver $(\mathrm{Ag})$ profile material is best profile material for designing the circular grating inside the fiber in terms of received optical power. The output power at the radius of $1.2 \mu \mathrm{m}$ of bragg cell is $7.56 \times 10^{-4} \mathrm{w} / \mathrm{m}^{2}$ and $\mathrm{It}$ is observed that the maximum received optical power at the output port is achieved $8.48 \times 10^{-4} \mathrm{w} / \mathrm{m}^{2}$ with $0.9 \mu \mathrm{m}$ radius of bragg cell and Silver (Ag) profile material at $0.8 \mathrm{w} / \mathrm{m}^{2}$ input transmission power. In practical applications, the Uniform fiber Bragg grating (U-FBG) is expected to be a key component of highly integrated photonic circuits like optical filter, splitters, optical sensor and switches etc. Thus for these applications, optimization of the designs and their complete simulations about material choice is important. To further increase the grating period, length of grating and reduction in fiber losses is still a challenging job demanding researchers to work out.

\section{ACKNOWLEDGMENTS}

Manjinder Kaur would like to thank Dr. Sanjeev Dewra at Shaheed Bhagat Singh State Technical campus (India) for valuable discussions.

\section{REFERENCES}

[1] F. Farahi, D.J. Webb and J.D.C. Jones, "Simultaneous measurement of temperature and strain: Cross-sensitivity considerations," J. Lightwave Technol., vol. 8, pp. 138148, Feb. 1990.

[2] J. Lopez-Higuera, "Handbook of Optical Fiber Sensing Technology,” Hoboken, NJ, USA: Wiley, 2002.

[3] A. Kersey et al., "Fiber grating sensors," J. Lightwave. Technol., vol. 15, no. 8, pp. 1442-1463, Aug. 1997.

[4] S. Y. Li, N. Q. Ngo, S. C. Tjin, P. Shum, and J. Zhang, "Thermally tunable narrow-bandpass filter based on a 
linearly chirped fiber Bragg grating," Opt. Lett., vol. 29, no. 1, pp. 29-31, Jan. 2004.

[5] H. Uno, A. Kojima, A. Shibano, and O. Mikami, "Optical wavelength switch using strain-controlled fiber Bragg gratings," in Proc. SPIE, 1999, pp. 274-277.

[6] J. Azana and M. Muriel, "Temporal self-imaging effects: Theory and application for multiplying pulse repetition rates,” IEEE J. Sel. Topics Quantum Electron., vol. 7, no. 4, pp. 728-744, Jul. 2001.

[7] M. M. Ali, K. S. Lim, H. Z. Yang, W. S. Chong, and H. Ahmad, B Direct period measurement for fiber Bragg grating using an optical imaging technique, Appl. Opt., vol. 52, no. 22, pp. 5393-5397, 2013.

[8] E.G. Turitsyna, J.D. Ania-Castanon, S.K. Turitsyn, L. Kennedy and K. Sugden, "Impact of design of sharp nonuniform fiber Bragg gratings on system performance", Electronics Letters, vol. 39,no. 4,pp 1-2. Feb 2003.

[9] Feced, R., Zervas, M.N., and Muriel, M.A.: 'An efficient inverse scattering algorithm for the design of non uniform fiber Bragg gratings', IEEE J.Quantum Electron., 1999, 35, pp. 1105.
[10] M. M. Ali, K. S. Lim, A. Becir, M. H. Lai and H. Ahmad, "Optical Gaussian Notch Filter Based on Periodic Micro bent Fiber Bragg Grating”IEEE Photonic Journal, vol. 6, no.1, February 2014.

[11] P. Niewczas, A. J. Willshire, L. Dziuda, and J. R. McDonald "Performance analysis of the Fiber Bragg Grating Interrogation System Based on an Arrayed Waveguide Grating", IEEE Transactions on Instrumentation and Measurement, vol. 53, no. 4, pp.1192-1196,August 2004.

[12] E.G. Turitsyna, J.D. Ania-Castanon, S.K. Turitsyn, L. Kennedy and K. Sugden, "Impact of design of sharp nonuniform fiber Bragg gratings on system performance", Electronics Letters, vol. 39,no. 4, pp 1-2. Feb 2003.

[13] Manpreet Singh, Sanjeev Dewra, R.S. Kaler, "Optimization and efficient routing scenario of system using C-band: Reconfigurable multi wavelength optical cross connect based on tunable fiber Bragg grating and optical circulator", Optical Engineering, Vol. 55, No.7, pp. 1-5, July 2016 